\title{
Curie temperatures of annealed FePt nanoparticle systems
}

\author{
Chuan-Bing Rong, Yang Li, and J. Ping Liu ${ }^{\mathrm{a})}$ \\ Department of Physics, University of Texas at Arlington, Arlington, Texas 76019
}

(Presented on 8 January 2007; received 26 October 2006; accepted 21 November 2006; published online 28 March 2007)

\begin{abstract}
The chemically synthesized $\mathrm{Fe}_{x} \mathrm{Pt}_{100-x}$ nanoparticles with controlled compositions were annealed to transfer the disordered face-centered-cubic structure to the ordered structure. It was found that the $L 1_{0} \mathrm{FePt}$ structure can be formed in the wide compositional region of $x=40-68$, and lower or higher $\mathrm{Fe}$ content leads to formation of the $\mathrm{L}_{2} \mathrm{FePt}_{3}$ or $\mathrm{Fe}_{3} \mathrm{Pt}$ phase, respectively. The Néel temperature of $L 1_{2} \mathrm{FePt}_{3}$ phase and Curie temperatures $\left(T_{C}\right)$ of $L 1_{0} \mathrm{FePt}$ and $L 1_{2} \mathrm{Fe}_{3} \mathrm{Pt}$ phases are all strongly composition dependent. The room-temperature saturation magnetization has an abnormal dependence on $x$ which is caused by the low $T_{C}$ of $\mathrm{Fe}_{3} \mathrm{Pt}$ phase with $x=75-79$. The big difference in $T_{C}$ between the heating and cooling thermomagnetic curves of the Fe-Pt alloys with $x=79-90$ can be attributed to the difference of $\alpha \Leftrightarrow \gamma$ phase transition temperature during heating and cooling. On the other hand, $T_{C}$ of the $L 1_{0} \mathrm{FePt}$ nanoparticles was found to be strongly size dependent.

(C) 2007 American Institute of Physics. [DOI: 10.1063/1.2709739]
\end{abstract}

\section{INTRODUCTION}

The interesting magnetic properties of the bulk $\mathrm{Fe}_{x} \mathrm{Pt}_{100-x}$ materials have been studied in the whole compositional region. For $x=25$ antiferromagnetic order was found; $;^{1,2}$ around $x=50$ the alloy shows very large magnetic anisotropy owing to the formation of chemically ordered face-centered-tetragonal (fct) structure. ${ }^{3,4}$ Near $x=75$ large volume instability (Invar effect) is observed in the $L 1_{2} \mathrm{Fe}_{3} \mathrm{Pt}$ phase $\left(\gamma_{1}\right)^{5,6}$ The most recent interests in FePt nanostructured materials have been focused on the ferromagnetic $L 1_{0}$ FePt phase $\left(\gamma_{2}\right)$ because of their potential applications in ultrahigh-density magnetic recording ${ }^{7,8}$ and advanced permanent magnets ${ }^{9,10}$ due to its very high uniaxial magnetocrystalline anisotropy and high Curie temperatures. Recently, it was reported that Curie temperature $\left(T_{C}\right)$ of sputtered $\mathrm{FePt}$ films is composition dependent in the range of 47.5-54.4 at. \% Fe. ${ }^{11-13}$ More interestingly, reduction of $T_{C}$ with decreasing dimension of the materials has also been observed in $L 1_{0} \mathrm{FePt}$ nanoparticles ${ }^{14}$ and other ferromagnets. ${ }^{15,16}$ In order to understand magnetic ordering of the nanostructured ferromagnetic phases in a wide compositional range, the annealed FePt nanocrystalline films deposited by nanoparticles have been studied with a composition range of $15-90$ at. $\% \mathrm{Fe}$. The size dependence of $T_{C}$ of $L 1_{0} \mathrm{FePt}$ nanoparticles has also been studied.

\section{EXPERIMENTS}

The disordered face-centered-cubic (fcc) $\mathrm{Fe}_{x} \mathrm{Pt}_{100-x}$ nanoparticles were chemically synthesized by the standard airless chemical solution procedures. ${ }^{7,17}$ The composition was controlled by adjusting the molar ratio of iron pentacarbonyl $\mathrm{Fe}(\mathrm{CO})_{5}$ to platinum acetylacetonate $\mathrm{Pt}(\mathrm{acac})_{2}$. The synthesized nanoparticles were then deposited on a Si substrate and annealed under forming gas $\left(\mathrm{Ar}+7 \% \mathrm{H}_{2}\right)$ at $973 \mathrm{~K}$

${ }^{a)}$ Electronic mail: pliu@uta.edu for $1 \mathrm{~h}$ to transfer the disordered fcc structure to the ordered $L 1_{0}$ or $L 1_{2}$ structure. The composition of the synthesized FePt nanoparticles was checked by energy dispersive x-ray (EDX) analysis. The crystalline structure was determined by $\mathrm{x}$-ray diffraction (XRD). The room-temperature magnetic properties were studied by a superconducting quantum interference device (SQUID) magnetometer with a maximum applied field of $7 \mathrm{~T}$. Thermomagnetic curves were measured by a physical property measurement system (PPMS) with hightemperature vibrating sample magnetometer.

\section{RESULTS AND DISCUSSIONS}

Figure 1 gives the composition dependence of the $4 \mathrm{~nm}$ synthesized $\mathrm{Fe}_{x} \mathrm{Pt}_{100-x}$ nanoparticles on the molar ratio of $\mathrm{Fe}(\mathrm{CO})_{5}$ to $\mathrm{Pt}(\mathrm{acac})_{2}$. The error bar is based on the statistical standard deviation of the EDX analysis. It shows that the composition $x=15-90$ can be obtained by adjusting the molar ratio of precursors. This result is similar to that of Ref. 17.

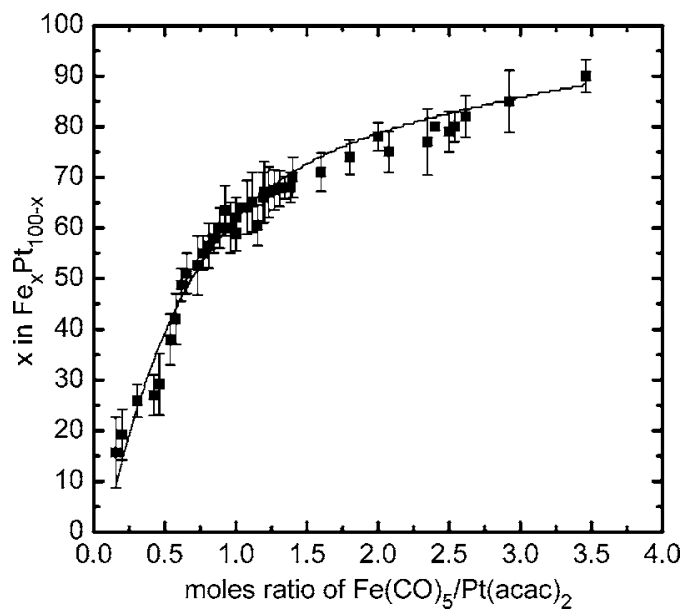

FIG. 1. The dependence of $x$ on the molar ratio of $\mathrm{Fe}(\mathrm{CO})_{5}$ to $\mathrm{Pt}(\mathrm{acac})_{2}$. 


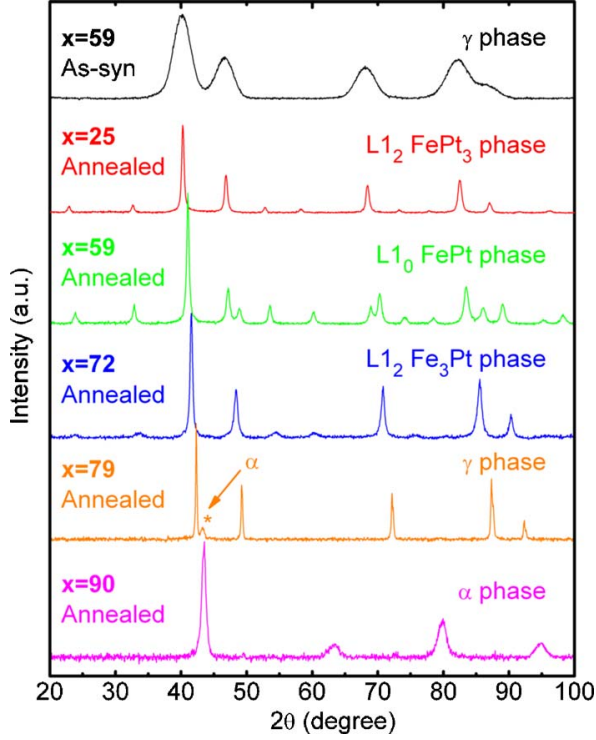

FIG. 2. (Color online) XRD patterns of the as-synthesized nanoparticles with $x=59$ and the annealed films with different compositions, i.e., $x=25$, 59, 72, 79, and 90 .

XRD patterns of $\mathrm{Fe}_{x} \mathrm{Pt}_{100-x}$ films were recorded after being annealed at $973 \mathrm{~K}$ in forming gas for $1 \mathrm{~h}$. Figure 2 shows the XRD patterns of the annealed films with five different compositions $x=25,59,72,79$, and 90 . A typical XRD pattern for the as-synthesized nanoparticles is also shown in the figure for comparison. It should be noted that XRD spectra of all the as-synthesized samples showed a disordered $\gamma$ phase in the whole compositional region. When the assembled films were annealed at $973 \mathrm{~K}$, on the other hand, $\mathrm{Fe}_{25} \mathrm{Pt}_{75}$ exhibits a $\mathrm{Cu}_{3} \mathrm{Au}$-type $\gamma_{3}-\mathrm{FePt}_{3}$ phase with $L 1_{2}$ structure. It was found that for the annealed $\mathrm{Fe}_{59} \mathrm{Pt}_{41}$ film the superlattice reflections (001) and (110) appear in the XRD spectra, indicating the formation of an ordered $L 1_{0} \gamma_{2}-\mathrm{FePt}$ phase. For $\mathrm{Fe}_{72} \mathrm{Pt}_{28}$, the annealing resulted in the formation of $L 1_{2} \gamma_{1}-\mathrm{Fe}_{3} \mathrm{Pt}$ phase. With increasing Fe content to $x=79$, XRD pattern revealed that the main phase was $\gamma$-FePt phase with fcc structure, while a small amount of $\alpha-(\mathrm{Fe}, \mathrm{Pt})$ was also found. Further increase of $\mathrm{Fe}$ content led to a decrease of $\gamma$-FePt phase content and an increase of $\alpha$-FePt phase content. When $x=90$, the annealed films consisted of only $\alpha$-FePt phase, as shown in Fig. 1. In general, the peak broadening of the annealed $\mathrm{Fe}_{x} \mathrm{Pt}_{100-x}$ films became less pronounced compared to the as-synthesized nanoparticles since the grains became coarse upon annealing. The average grain size was $4 \mathrm{~nm}$ for the as-synthesized nanoparticles, while it was around 10-20 nm after annealing on Si substrates, determined by the analysis of XRD patterns using Scherrer formula.

Figure 3 shows the dependence of Néel temperature $T_{N}$ or Curie temperature $T_{C}$ on composition of the annealed nanoparticles. The magnetic phase transition temperatures were determined by the intersection of extrapolations of the greatest slope and flat region in the $M-T$ curves. It was reported that $\mathrm{FePt}_{3}$ is antiferromagnetic in the chemically ordered state. ${ }^{1,2}$ The XRD patterns show that the ordered phase $\mathrm{FePt}_{3}$ with $\mathrm{Cu}_{3} \mathrm{Au}$ cubic $L 1_{2}$ structure can be obtained when $x \leqslant 40$, which is similar to the condition shown in the phase

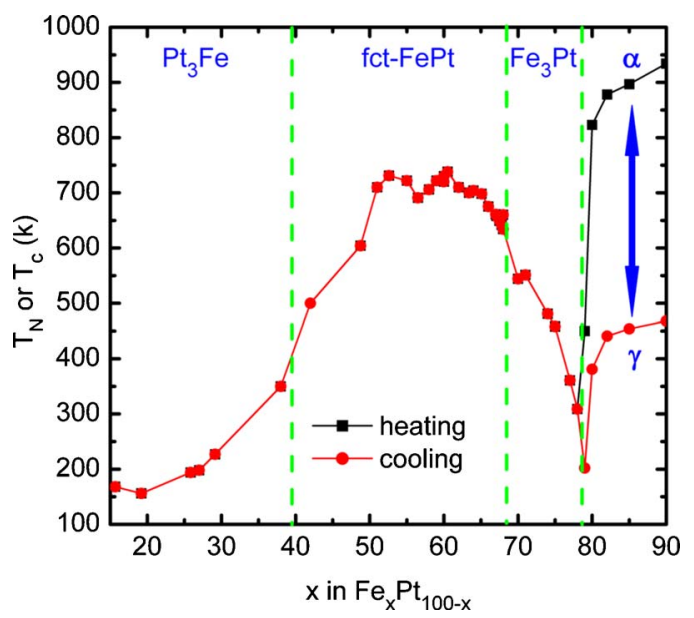

FIG. 3. (Color online) The dependence of $T_{N}$ or $T_{C}$ on $x$ of the annealed nanoparticles.

diagram. ${ }^{18}$ It was found that $T_{N}$ of the $\mathrm{FePt}_{3}$ phase increased with increasing Fe content. An ordered fct FePt ferromagnetic phase with $L 1_{0}$ structure formed within the compositional region $x=40-69 . T_{C}$ of the fct phase increased fast when $x \leqslant 50$ and reached the maximum about $730 \mathrm{~K}$ when $x=51-64$ and then dropped with increasing $x$. For $69 \leqslant x$ $\leqslant 78, \mathrm{Fe}_{3} \mathrm{Pt}$ phase with $L 1_{2}$ structure was formed after annealing. It can be seen that $T_{C}$ decreased dramatically from $550 \mathrm{~K}$ with $x=70$ to $310 \mathrm{~K}$ with $x=79$. More interestingly, it was found that there is a big difference between the heating and cooling thermomagnetic curves of the $\mathrm{Fe}-\mathrm{Pt}$ alloys with Fe content of 80-90 at. \%. Figure 4 shows the typically heating and cooling $M-T$ curves of the $\mathrm{Fe}_{80} \mathrm{Pt}_{20}$ sample. This phenomenon can be attributed to the difference of $\alpha \Leftrightarrow \gamma$ phase transition temperature during heating and cooling. ${ }^{19}$ One can see from the Fe-Pt phase diagram ${ }^{18}$ that the phase transitional temperature from $\alpha$ to $\gamma$ phase on heating is far higher than that of $\gamma$ to $\alpha$ on cooling.

Figure 5 gives the dependence of saturation magnetization $M_{s}$ and coercivity $H_{c}$ on the composition of annealed nanoparticles. $M_{s}$ at room temperature, which was measured under $7 \mathrm{~T}$, increases monotonously almost in the whole compositional region except in the region $75<x \leqslant 79$ where $M_{s}$ of the $\mathrm{Fe}_{3} \mathrm{Pt}$ phase drops. This is caused by the low Curie

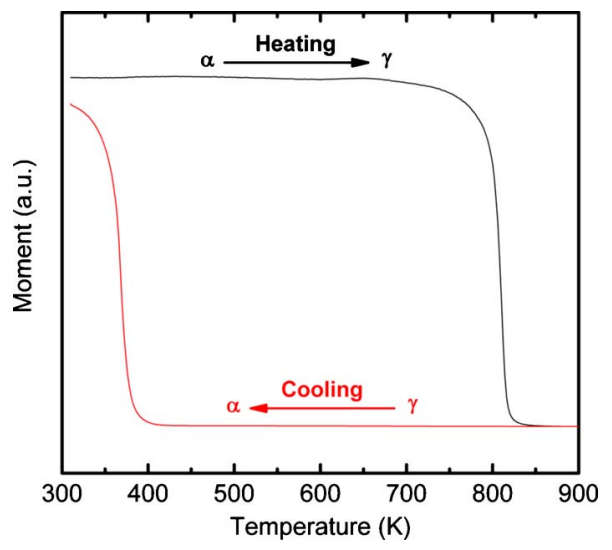

FIG. 4. (Color online) The typically heating and cooling $M-T$ curves of the $\mathrm{Fe}_{80} \mathrm{Pt}_{20}$ sample. 


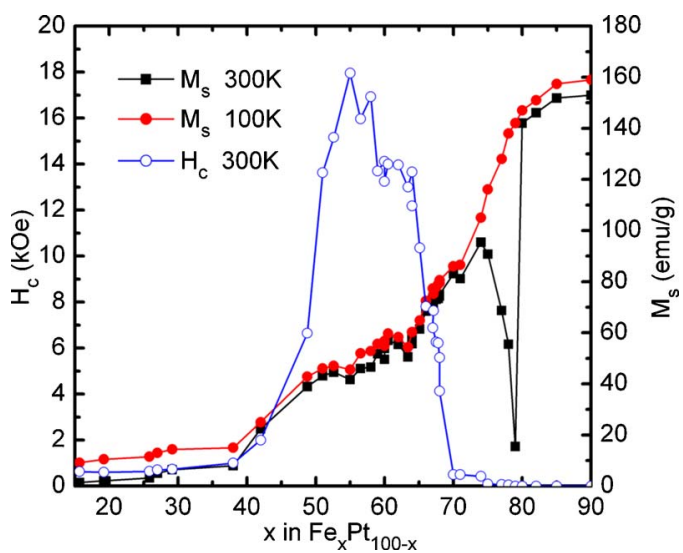

FIG. 5. (Color online) The dependence of $M_{s}$ and $H_{c}$ on $x$ of annealed nanoparticles.

temperature (near or lower than room temperature) of $\mathrm{Fe}_{3} \mathrm{Pt}$ phase in this special compositional region since there is no dip in the $M_{s}-x$ curve at $100 \mathrm{~K}$, as shown in Fig. $5 . H_{c}$ is also very sensitive to composition. Nonzero coercivity can be obtained only in the region $x=42-68$ where $L 1_{0}$ phase was formed. The maximum $H_{c}$ is $18 \mathrm{kOe}$ at $x=55$. With both high $M_{s}$ and $H_{c}$, the maximum energy product $(B H)_{\max }$ about $17 \mathrm{MG}$ Oe can be obtained at $x=66$ where $H_{c}=7.6 \mathrm{kOe}$.

Beside the composition dependence, it was also found that $T_{C}$ decreases fast with decreasing dimensions of the materials. Our recently developed salt-matrix annealing method $^{20}$ allows us to tune the size of the monodisperse $L 1_{0}$ FePt particles and to obtain a direct correlation between particle size and Curie temperature. Figure 6 shows $M-T$ curves of the 3 and $15 \mathrm{~nm} L 1_{0}$ FePt nanoparticles which are obtained by the salt-matrix. It shows that $T_{C}$ of the $3 \mathrm{~nm}$ particles is substantially lower than that of $15 \mathrm{~nm}$ particles. A systematic study of the size dependence of $T_{C}$ has been reported elsewhere. ${ }^{14}$ The size dependent behavior, as discussed in Refs. 14-16, can be explained by the finite-sizescaling theory. ${ }^{21,22}$

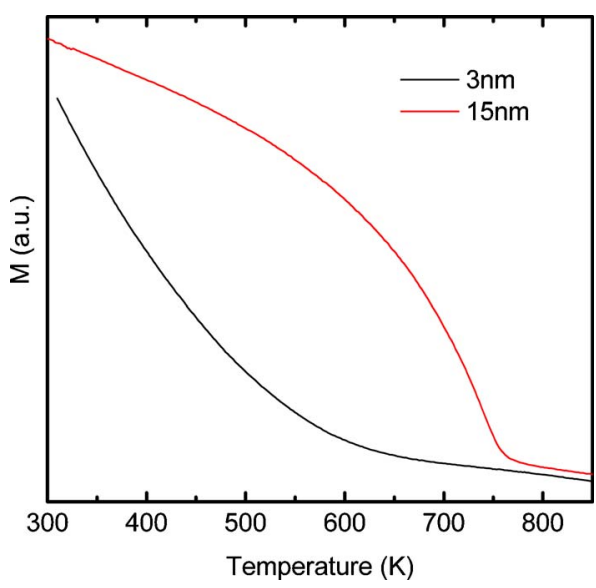

FIG. 6. (Color online) $M-T$ curves of the 3 and $15 \mathrm{~nm} L 1_{0}$ nanoparticles which are obtained by the salt-matrix method.

\section{CONCLUSION}

The FePt nanoparticles with controlled composition have been prepared by chemical synthesis. The ordered phases with different structures were obtained by annealing the particles at $973 \mathrm{~K}$ for $1 \mathrm{~h}$. It is found that the $L 1_{0} \mathrm{FePt}$ structure can be formed in a wide compositional region of $x=40-68$, while the $\mathrm{FePt}_{3}$ or $\mathrm{Fe}_{3} \mathrm{Pt}$ with $L 1_{2}$ structure are formed with lower or higher Fe contents. The Curie temperatures of the $L 1_{0}$ FePt phase are strongly composition dependent and are higher than that of $\mathrm{Fe}_{3} \mathrm{Pt}$ phase. The abnormal dependence of $M_{s}$ at room temperature on $x$ is caused by the low $T_{C}$ of $\mathrm{Fe}_{3} \mathrm{Pt}$ phase with $x=75-79$. It was also found that there is a big difference in $T_{C}$ when measured through the heating and cooling thermomagnetic curves of the Fe-Pt alloys with $x$ $=78-90$. This phenomenon can be attributed to the difference of $\alpha \Leftrightarrow \gamma$ phase transition temperature during heating and cooling. On the other hand, $T_{C}$ of the $L 1_{0}$ FePt nanoparticles is strongly size dependent.

\section{ACKNOWLEDGMENTS}

This work was supported by US DoD/MURI Grant No. N00014-05-1-0497 and DARPA through ARO under Grant No. DAAD 19-03-1-0038.

${ }^{1}$ G. E. Bacon and J. Crangle, Proc. R. Soc. London, Ser. A 272, 387 (1963). ${ }^{2}$ S. Maat, O. Hellwig, G. Zeltzer, E. E. Fullerton, G. J. Mankey, M. L. Crow, and J. L. Robertson, Phys. Rev. B 63, 134426 (2001).

${ }^{3}$ H. Kanazawa, G. Lauhoff, and T. Suzuki, J. Appl. Phys. 87, 6143 (2000).

${ }^{4}$ S. Okamoto, N. Kikuchi, O. Kitakami, T. Miyazaki, Y. Shimada, and K. Fukamichi, Phys. Rev. B 66, 024413 (2002).

${ }^{5}$ R. Hayn and V. Drchal, Phys. Rev. B 58, 4341 (1998).

${ }^{6}$ M. Matsushita, Y. Miyoshi, S. Endo, and F. Ono, Phys. Rev. B 72, 214404 (2005)

${ }^{7}$ S. H. Sun, C. B. Murray, D. Weller, L. Folks, and A. Moser, Science 287, 1989 (2000).

${ }^{8}$ C. Ross, Annu. Rev. Mater. Res. 31, 203 (2001).

${ }^{9}$ H. Zeng, J. Li, J. P. Liu, Z. L. Wang, and S. H. Sun, Nature (London) 420 , 395 (2002)

${ }^{10}$ C. B. Rong, H. W. Zhang, X. B. Du, J. Zhang, S. Y. Zhang, and B. G. Shen, J. Appl. Phys. 96, 3921 (2004).

${ }^{11}$ K. Barmak, J. Kim, S. Shell, E. B. Svedberg, and J. K. Howard, Appl. Phys. Lett. 80, 4268 (2002).

${ }^{12}$ K. Barmak, J. Kim, D. C. Berry, K. Wierman, E. Svedberg, and J. K. Howard, J. Appl. Phys. 95, 7486 (2004).

${ }^{13}$ K. Barmak, J. Kim, D. C. Berry, W. N. Hanani, K. Wierman, E. B. Svedberg, and J. K. Howard, J. Appl. Phys. 97, 024902 (2005).

${ }^{14}$ C. B. Rong, D. R. Li, V. Nandwana, N. Poudyal, Y. Ding, Z. L. Wang, H. Zeng, and J. P. Liu, Adv. Mater. (Weinheim, Ger.) 18, 2984 (2006).

${ }^{15}$ M. Farle, K. Baberschke, U. Stetter, A. Aspelmeier, and F. Gerhardter, Phys. Rev. B 47, 11571 (1993).

${ }^{16}$ J. P. Chen, C. M. Sorensen, K. J. Klabunde, and G. C. Hadjipanayis, Phys. Rev. B 54, 9288 (1996).

${ }^{17}$ S. H. Sun, E. E. Fullerton, D. Weller, and C. B. Murray, IEEE Trans. Magn. 37, 1239 (2001).

${ }^{18} \mathrm{M}$. Hausen and K. Anderko, Constitution of Binary Alloys (McGraw-Hill, New York, 1958).

${ }^{19}$ J. Martelly, Ann. Phys. (N. Y.) 9, 318 (1938).

${ }^{20}$ K. Elkins, D. Li, N. Poudyal, V. Nandwana, Z. Q. Jin, K. H. Chen, and J. P. Liu, J. Phys. D 38, 2306 (2005).

${ }^{21}$ K. Binder, Physica (Amsterdam) 62, 508 (1972).

${ }^{22}$ S. N. Kaul, J. Magn. Magn. Mater. 53, 5 (1985). 